\title{
Nutrition in the palliative phase of Parkinson's disease: a scoping review of Parkinson's guidelines for medical and allied health professionals
}

\author{
M.Ó. Breasail ${ }^{1}$, S.E. Goldsmith ${ }^{2}$, E.J. Henderson ${ }^{1,3}$ and F.E. Lithander ${ }^{1}$ \\ ${ }^{1}$ Population Health Sciences, Bristol Medical School, University of Bristol, Bristol, UK, \\ ${ }^{2}$ Translational Health Sciences, Bristol Medical School, University of Bristol, Bristol, UK and \\ ${ }^{3}$ Royal United Hospital Bath NHS Foundation Trust, Bath, UK
}

Objective: To summarise the extent to which nutrition in the palliative phase of Parkinson's disease is addressed in Parkinson's disease guidelines for medical and allied health professionals. Parkinson's disease is a heterogenous condition, people with Parkinson's (PwP) are susceptible to weight loss and malnutrition. This results from increased energy expenditure due to dyskinesia (involuntary movements) and the deleterious effects of disease symptoms and medication side-effects on nutrient intake and metabolism. Dysphagia is common in advanced disease. Increasing disease severity may indicate the need for clinically-assisted nutrition and hydration $(\mathrm{CANH})$, though evidence of benefit is not established. Whether nutrition in the palliative phase of Parkinson's disease can prolong life and/or improve quality of life is an important consideration in the care of PwP. Guidelines for clinical practice, nutrition and palliative care can help to simplify and prioritise the management of complex needs.

A scoping review was performed using PUBMED, CINAHL, AMED, EMBASE, PsycINFO, Google Scholar, and Google Search between 22/02/21 and 16/03/21. Search terms were 'practice guidelines Parkinson's Disease', 'nutrition guidelines Parkinson's Disease', 'palliative care Parkinson's Disease guidelines'. Searches further specified "English language” and in "humans" where possible.

Eighteen guidelines were identified as containing Parkinson's-specific guidance relating to food, diet, or nutrition aimed at medical, nursing, or allied health professionals. Sixteen of these were Parkinson's-specific; 1 focused on clinical nutrition in neurology contained extensive Parkinson's-specific guidance and another focused on CANH in adults without the capacity to consent contained limited Parkinson's-specific advice. Guidance relating to diet and nutrition was most frequently provided in the context of dysphagia $(16 / 18)$ or in relation to the effects of dietary protein on levodopa metabolism (13/18). CANH was discussed in 6/18 guidelines. Nutrition in the palliative phase of Parkinson's was discussed in 3/18 publications. However, only 2 out of these 3 were Parkinson's-specific and were aimed at healthcare professionals and dieticians respectively: The Irish Palliative Care in Parkinson's Disease Group 2016 guidelines $^{(1)}$ and the British Dietetic Association/Parkinson's UK 2021 guidelines ${ }^{(2)}$. Both highlight the importance of early planning for more advanced disease stages, in addition to specific guidance related to the withdrawal of CANH if it causes patient distress or reduces remaining quality of life.

PwP have complex individual healthcare needs and it may be difficult to give generalised recommendations for nutrition in the palliative phase of Parkinson's disease. Current guidelines for medical and allied health professionals focus mostly on the management of the specific symptoms of dysphagia and constipation. In relation to planning nutrition in the palliative stage of Parkinson's disease, the importance of early decision making in relation to CANH is emphasised whereby benefits and burdens can be weighed up to reach a decision that maximises an individual's quality of life.

\section{References}

1. The Irish Palliative Care in Parkinson's Disease Group. (2016).

2. British Dietetic Association. (2021). 